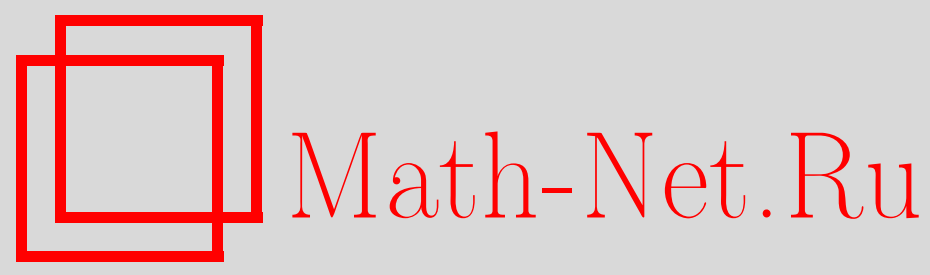

П. В. Довбуш, О существовании $K$-пределов голоморфных отображений, Матем. заметки, 2005, том 77, выпуск 4, 509-514

DOI: https://doi.org/10.4213/mzm2509

Использование Общероссийского математического портала Math-Net.Ru подразумевает, что вы прочитали и согласны с пользовательским соглашением http://www.mathnet.ru/rus/agreement

Параметры загрузки:

IP : 54.198 .187 .58

26 апреля 2023 г., 12:59:26

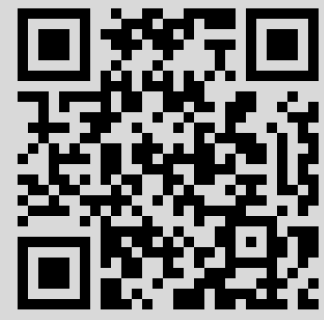




\section{О СУЩЕСТВОВАНИИ $K$-ПРЕДЕЛОВ ГОЛОМОРФНЫХ ОТОБРАЖЕНИЙ}

\section{П. В. Довбуш}

Пусть $D$ - полная гиперболическая область в $\mathbb{C}^{n}, n>1$, а $N$ - компактное эрмитово многообразие. Для произвольного голоморфного отображения $f: D \rightarrow N$ в произвольнй граничной точке $D$ доказьвается критерий существования $K$-предела у $f$ при условии, что соответствующий радиальный предел у $f$ в этой точке существует.

Библиографоия: 11 названий.

Если $U$ - единичный круг в $\mathbb{C}$, а $M$ - комплексное многообразие комплексной размерности $n, p$ - его точка, а $v \in T_{p} M-$ касательньй вектор, то полунорма Кобаяси

$$
K_{M}(p, v)=\inf \left\{\frac{1}{\lambda} \mid h \in H(U, M), \quad h(0)=p, \quad h^{\prime}(0)=\lambda \cdot v, \quad \lambda>0\right\},
$$

а расстояние между точками $p, q \in M$

$$
k_{M}(p, q)=\inf _{\gamma} \int_{0}^{1} K_{M}\left(\gamma(t), \gamma^{\prime}(t)\right) d t
$$

где нижняя грань берется по всем гладким путям $\gamma:[0,1] \rightarrow M$, соединяющим точки $p$ и $q$.

$\mathrm{X}$. Ройден [1] доказал, что полуметрика $k_{D}$ совпадает с полуметрикой, введенной Кобаяси [2], и что понятие гиперболического многообразия, также введенное ранее Кобаяси, может быть определено в терминах нормы Кобаяси: многообразие $M$ гиперболично тогда и только тогда, когда для каждой точки $p \in M$ существует координатная окрестность $W$ и положительная постоянная $c$ такая, что для всех $(q, v) \in T(W) \simeq W \times \mathbb{C}^{n}$, $T(W)=\cup_{q \in W} T_{q}(M)$,

$$
K_{M}(q, v) \geqslant c \cdot\|v\|,
$$

где $\|\cdot\|$ - евклидова норма в $\mathbb{C}^{n}$.

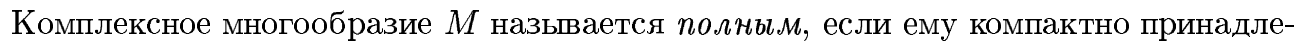
жит любой шар в метрике Кобаяси. Известно (см. [3]), что полными гиперболическими многообразиями являются все ограниченные области голоморфности в $\mathbb{C}^{n}$ с достаточно гладкими гранищами.

Пусть $H(M, N)$ - множество всех голоморфных отображений комплексного многообразия $M$ в комплексноемногообразие $N$. Последовательность $\left\{f_{j}\right\} \subset H(M, N)$ назьвается компактно расходящейся, если для любых компактов $K \subset M$ и $K^{\prime} \subset N$ найдется 
$j_{0}$ такое, что $f_{j_{0}}(K) \cap K^{\prime}=\varnothing$ для всех $j>j_{0}$. Семейство $\mathscr{F} \subset H(M, N)$ называется нормальным м, если из любой последовательности $\left\{f_{j}\right\} \subset \mathscr{F}$ можно извлечь подпоследовательность $\left\{f_{j_{0}}\right\}$ либо равномерно сходящуюся на компактных подмножествах $M$, либо компактно расходящуюся.

Комплексное многообразие $M$ называется упругим, если нормально семейство $H(U, M)$. Любое полное гиперболическое многообразие упруго (см. [4]). Если $M$ упруго, то нетрудно видеть, что нижняя грань в (1) всегда достигается для некоторого $h \in H(U, M)$.

Пусть всюду в дальнейшем $D$ - полная гиперболическая область в $\mathbb{C}^{n}$, а $N$ - эрмитово комплексное многообразие с эрмитовой метрикой $d s_{N}^{2}$. Расстояние, связанное с метрикой $d s_{N}^{2}$, будем обозначать через $s_{N}$, а через $d s_{N}(a, u)$ будем обозначать $\sqrt{d s_{N}^{2}(a, u)}-$ норму касательного вектора $u \mathrm{~K} N$ в точке $a \in N$.

Предположим, что в точке $\xi \in \partial D$ сушествует внешняя нормаль $\nu_{\xi}$. Обозначим

$$
N_{\xi}=\left\{\xi-t \nu_{\xi} \in D: 0<t \leqslant \delta\right\} \text {. }
$$

Отметим, что $N_{\xi}-$ множество точек из $D$, принадлежащих отрезку евклидовой длины $\delta$, перпендикулярному к касательной плоскости к $\partial D$ в точке $\xi$.

Будем говорить, что отображение $f: D \rightarrow N$ имеет в точке $\xi \in \partial D$ радиальньй предел $l \in N$, если

$$
\lim _{t \rightarrow 0} s_{N}\left(f\left(\xi-t \nu_{\xi}\right), l\right)=0 .
$$

$K$-допустимой областью раствора $\beta>0$ в точке $\xi \in \partial D$ (см. [5]) называется область

$$
K_{\beta}(\xi)=\left\{z \in D: k_{D}\left(z, N_{\xi}\right)<\beta\right\}
$$

Здесь и далее $k_{D}\left(z, N_{\xi}\right)=\inf \left\{k_{D}(z, w), w \in N_{\xi}\right\}$.

Введем следующий вариант понятия $K$-предела: будем говорить, что отображение $f: D \rightarrow N$ имеет $K$-пределl $\in N$ в точке $\xi \in \partial D$, т.е.

$$
(K-\lim f)(\xi)=l,
$$

если для любого $\beta>0$ и для любой последовательности точек $\left\{z_{j}\right\}$ из $K_{\beta}(\xi)$, сходяшейся к $\xi, s_{N}\left(f\left(z_{j}\right), l\right) \rightarrow 0$ при $j \rightarrow \infty$.

Чтобы установить связь между существованием радиального и $K$-пределов у произвольного отображения $f \in H(D, N)$ обозначим

$$
Q_{f}(z)=\sup _{v \in \mathbb{C}^{n} \backslash\{0\}}\left\{\frac{d s_{N}\left(f(z), f_{*}(v)\right)}{K_{D}(z, v)}\right\}
$$

где $f_{*}=d f-$ дифференциал отображения $f$ в точке $z \in D$.

Пусть $Y$ - относительно компактное комплексное подпространство эрмитового многообразия $N$. Символом $H(D, Y)$ будем обозначать подмножество $H(D, N)$ такое, что $f(D) \subset Y$. 
Теорема 1. Пусть $Y$ - относительно компактное комплексное подпространство әрмитова многообразия $N$ с әрмитовой метрикой $d s_{N}^{2}$, а $D$ - полная гиперболическая область в $\mathbb{C}^{n}, n>1$, имеющая нормаль в точке $\xi \in \partial D$. Если отображсение $f \in H(D, Y)$ имеет в точке $\xi$ радиальный предел $l \in \bar{Y}$, то $f$ в точке $\xi$ имеет $K$-предел тогда и только тогда, когда

$$
\left(K-\lim Q_{f}\right)(\xi)=0 .
$$

Лехто и Виртанен [6] доказали теорему, согласно которой если мероморфная в единичном круге $U$ функция $f$ имеет в точке $1 \in \partial U$ радиальный предел, то $f$ имеет в этой точке угловой предел тогда и только тогда, когда в каждом угле $|\arg (1-z)|<\pi / 2-\delta$, где $\delta$ - любое положительное число, вьполняется соотношение

$$
\left|Q_{f}(z)\right|=O(1)
$$

где $O(1)$ - ограниченная функция.

В случае многих комплексных переменных ограниченность функции $\left|Q_{f}(z)\right|$ даже на всей области определения отображения $f$ не гарантирует существования $K$-предела у $f$ в произвольной граничной точке, в которой у $f$ существует радиальньй предел.

В самом деле, функция $f(z)=\left(z_{2}\right)^{2} /\left(1-z_{1}\right)$ ограничена и голоморфна в единичном шаре $B=\left\{z \in \mathbb{C}^{2},|z|<1\right\}$.

В случае единичного шара норма Кобаяси совпадает с нормой Каратеодори; отсюда и из определения нормы Каратеодори следует, что

$$
\left|f_{*}(v)\right| \leqslant \sup \{|f(z)|, z \in B\} \cdot K_{B}(z, v)
$$

для всех $z \in B$ и всех $v \in \mathbb{C}$, поэтому

$$
\left|Q_{f}(z)\right| \leqslant \sup \{|f(z)|, z \in B\} .
$$

Однако функция $f$ имеет в точке $(1,0) \in \partial B$ радиальный предел, равньй нулю, и предел, равный единище, по точкам $\left(1-2^{-2 m}, 2^{-m}\right)$, заведомо принадлежащим множеству $K_{50}((1,0))$. Следовательно, функция $f$ не может иметь $K$-предела в точке $(1,0)$.

ДОКАЗАТЕЛЬСТВО ТЕОРЕМЫ 1. ПредПоложим, что $f$ имеет радиальный предел $l \in \bar{Y}$ в точке $\xi$ и выполняется (2). Покажем, что тогда $f$ имеет $K$-предел $l$ в точке $\xi$. По определению $K$-предела для этого надо показать, что для любого фиксированного $\beta$ и для любой последовательности $\left\{z_{j}\right\} \subset K_{\beta}(\xi)$ такой, что $z_{j} \rightarrow \xi$ при $j \rightarrow \infty$,

$$
\lim _{j \rightarrow \infty} s_{N}\left(f\left(z_{j}\right), l\right)=0 .
$$

Фиксируем произвольное $\beta>0$. Так как $D$-полная гиперболическая область, ееможно представить в виде

$$
D=\bigcup_{j=1}^{\infty} D_{j}
$$

где

$$
D_{j}=\left\{z \in D: k_{D}\left(z, z_{0}\right) \leqslant \beta \cdot j\right\}, \quad j=1,2, \ldots,
$$


а $z_{0}$ - некоторая точка, принадлежащая $N_{\xi} \subset D$.

Пусть $\left\{z_{j}\right\}$ - произвольная последовательность точек из $K_{\beta}(\xi)$ такая, что $z_{j} \rightarrow \xi$ при $j \rightarrow \infty$, и пусть $\left\{w_{j}\right\}$ - такая последовательность точек из $N_{\xi}$, что $k_{D}\left(w_{j}, z_{j}\right)=$ $k_{D}\left(z_{j}, N_{\xi}\right)$. Тогда из определения множества $K_{\beta}(\xi)$ следует, что $k_{D}\left(z_{j}, w_{j}\right) \leqslant \beta$.

Предположим, что $z_{j} \in D_{k_{j}+1} \backslash D_{k_{j}}$ для некоторого $k_{j} \in \mathbb{N}$. Так как $D-$ полная гиперболическая область, то шары в метрике Кобаяси $\left\{z \in D: k_{D}\left(z_{0}, z\right)<\beta \cdot j\right\}$ компактно принадлежат $D$, и поскольку $z_{j} \rightarrow \xi$ при $j \rightarrow \infty$, то числа $k_{j} \rightarrow \infty$ при $j \rightarrow \infty$.

Из неравенства треугольника для метрики Кобаяси получаем

$$
k_{D}\left(w_{j}, z_{0}\right) \geqslant k_{D}\left(z_{j}, z_{0}\right)-k_{D}\left(z_{j}, w_{j}\right)>\beta \cdot\left(k_{j}-1\right) .
$$

Следовательно, $w_{j} \in N_{\xi} \cup\left(D \backslash D_{k_{j}-1}\right)$ и, значит, $w_{j} \rightarrow \xi$ при $j \rightarrow \infty$.

Так как вьполняется (2), то величины

$$
\epsilon_{j}=\sup \left\{Q_{f}(z): z \in K_{\beta}(\xi) \cap\left(D \backslash D_{j}\right)\right\}
$$

существуют, конечны для каждого $j$ и стремятся к нулю при $j \rightarrow \infty$. Поэтому

$$
s_{N}\left(f\left(z_{j}\right), f\left(w_{j}\right)\right) \leqslant k_{D}\left(z_{j}, w_{j}\right) \cdot \epsilon_{k_{j}-1} \leqslant \beta \cdot \epsilon_{k_{j}-1}
$$

и, значит,

$$
\lim _{j \rightarrow \infty} s_{N}\left(f\left(z_{j}\right), f\left(w_{j}\right)\right)=0 .
$$

Из неравенства треугольника для метрики $s_{N}$ получаем

$$
s_{N}\left(l, f\left(z_{j}\right)\right) \leqslant s_{N}\left(f\left(w_{j}\right), l\right)+s_{N}\left(f\left(z_{j}\right), f\left(w_{j}\right)\right) .
$$

Первое слагаемое в правой части (3) может быть сделано сколь угодно малым, поскольку $\left\{w_{j}\right\} \subset N_{\xi}, w_{j} \rightarrow \xi$ при $j \rightarrow \infty$ и по условию $f$ имеет радиальный предел $l$ в точке $\xi$. Второе слагаемое в правой части (3) также может быть сделано сколь угодно малым, так как по доказанному выше $\lim _{j \rightarrow \infty} s_{N}\left(f\left(z_{j}\right), f\left(w_{j}\right)\right)=0$. Поэтому из (3) следует, что $\lim _{j \rightarrow \infty} s_{N}\left(f\left(z_{j}\right), l\right)=0$.

Докажем обратное утверждение. Предположим, что $f$ имеет $K$-предел $l \in \bar{Y}$ в точке $\xi$; надо показать, что вьполняется неравенство $(2)$. Если $f$ имеет $K$-предел $l$ в точке $\xi$, то нетрудно видеть, что для каждого $\epsilon$ существует $k_{j} \in \mathbb{N}$ такое, что

$$
s_{N}(f(z), l) \leqslant \epsilon
$$

для всех $z \in K_{\beta}(\xi) \cap\left(D \backslash D_{k_{j}}\right)$.

Для того чтобы показать, что вьполняется (2), надо показать, что

$$
\lim _{j \rightarrow \infty} Q_{f}\left(z_{j}\right)=0
$$

для любого $\beta>0$ и любой последовательности $\left\{z_{j}\right\} \subset K_{\beta / 2}(\xi)$, сходящейся к $\xi$. Фиксируем произвольное $\beta>0$ и произвольный вектор $v \in \mathbb{C}^{n} \backslash\{0\}$. Выберем любую последовательность $\left\{z_{j}\right\} \subset K_{\beta / 2}(\xi)$ такую, что $z_{j} \rightarrow \xi$ при $j \rightarrow \infty$. Так как $D-$ полная гиперболическая область, для каждой точки $z_{j}$ существует отображение $h_{j} \in H(U, D)$ 
такое, что $h_{j}(0)=z_{j}$ и $h_{j}^{\prime}(0)=v / K_{D}\left(z_{j}, v\right)$. Поскольку $h_{j}$ голоморфно отображает $U$ в $D$, по свойству сжимаемости метрики Кобаяси для всех $\lambda \in U$

$$
k_{D}\left(h_{j}(0), h_{j}(\lambda)\right) \leqslant k_{U}(0, \lambda)
$$

а так как $h_{j}(0)=z_{j} \in K_{\beta / 2}(\xi)$, по определению множества $K_{\beta / 2}(\xi)$

$$
k_{D}\left(z_{j}, N_{\xi}\right)=k_{D}\left(h_{j}(0), N_{\xi}\right)<\frac{\beta}{2} .
$$

Из (5) и (6) и неравенства треугольника для метрики Кобаяси заключаем, что для всех $\lambda \in d_{\beta / 2}=\left\{\lambda \in U: k_{U}(0, \lambda)<\beta / 2\right\}$ имеют место следуюшие неравенства:

$$
\begin{gathered}
k_{D}\left(h_{j}(\lambda), N_{\xi}\right) \leqslant k_{D}\left(h_{j}(\lambda), z_{j}\right)+k_{D}\left(z_{j}, N_{\xi}\right) \leqslant \frac{\beta}{2}+\frac{\beta}{2}=\beta, \\
k_{D}\left(h_{j}(\lambda), z_{0}\right) \geqslant k_{D}\left(h_{j}(0), z_{0}\right)-k_{D}\left(h_{j}(0), h_{j}(\lambda)\right) \geqslant k_{D}\left(z_{j}, z_{0}\right)-\frac{\beta}{2} .
\end{gathered}
$$

Из (7) следует, что для всех $j$ имеет место включение

$$
h_{j}\left(d_{\beta / 2}\right) \subset K_{\beta}(\xi)
$$

Предположим теперь, что $z_{j} \in D_{k_{j}+1} \backslash D_{k_{j}}$; тогда

$$
\beta \cdot k_{j} \leqslant k_{D}\left(z_{j}, z_{0}\right) \leqslant \beta \cdot\left(k_{j}+1\right)
$$

и из $(8)$ следует, что $k_{D}\left(h_{j}(\lambda), z_{0}\right) \geqslant \beta \cdot\left(k_{j}-1 / 2\right) \geqslant \beta \cdot\left(k_{j}-1\right)$, поэтому

$$
h_{j}\left(d_{\beta / 2}\right) \subset\left(D \backslash D_{k_{j}}\right)
$$

Из $(9)$ и $(10)$ следует, что существует $J=J\left(k_{j}\right)$ такое, что $h_{j}\left(d_{\beta / 2}\right) \subset K_{\beta}(\xi) \cap\left(D \backslash D_{k_{j}}\right)$ для всех $j \geqslant J$. Отсюда и из (4) получаем, что

$$
s_{N}\left(f\left(h_{j}(\lambda), l\right) \leqslant \epsilon\right.
$$

для всех $\lambda \in d_{\beta / 2}, j \geqslant J$. Отсюда следует, что семейство $\mathscr{F}=\left\{f \circ h_{j}, j \geqslant J\right\}$ равностепенно непрерьвно и, следовательно, нормально в $d_{\beta / 2}$, так как $\bar{Y}$ компактно, и, значит, отображения $f \circ h_{j}, j \geqslant J$, сходятся равномерно на $d_{\beta / 2}$ к постоянному отображению. Из одномерной теоремы Вейерштрасса следует, что $f_{j}^{\prime}(0) \rightarrow 0$, поэтому

$$
\lim _{j \rightarrow \infty} d s_{N}\left(f_{j}(0), f_{j}^{\prime}(0)\right)=0
$$

Так как $h_{j}^{\prime}(0)=v / K_{D}\left(z_{j}, v\right)$, прямые вычисления показьвают, что

$$
d s_{N}\left(f_{j}(0), f_{j}^{\prime}(0)\right)=\frac{d s_{N}\left(f\left(z_{j}\right), f_{*}(v)\right)}{K_{D}\left(z_{j}, v\right)} .
$$


Следовательно,

$$
\lim _{j \rightarrow \infty} \frac{d s_{N}\left(f\left(z_{j}\right), f_{*}(v)\right)}{K_{D}\left(z_{j}, v\right)}=0 .
$$

Поскольку последовательность $\left\{z_{j}\right\} \subset \mathscr{K}_{\beta / 2}(\xi)$ и вектор $v \in \mathbb{C}^{n} \backslash\{0\}$ были выбраны произвольно, из (11) следует, что $\lim _{j \rightarrow \infty} Q_{f}\left(z_{j}\right)=0$ для любой последовательности $\left\{z_{j}\right\} \subset K_{\beta / 2}(\xi)$ такой, что $z_{j} \rightarrow \xi$ при $j \rightarrow \infty$. Отсюда в силу произвольности $\beta$ заключаем, что отображение $f$ имеет $K$-предел в точке $\xi$.

Теорема доказана.

Строго псевдовьпуклые области в $\mathbb{C}^{n}$ являются полными гиперболическими, а нормы Бергмана и Кобаяси таких областей имеют одинаковое граничное поведение (см. [7], [8]), поэтому в случае строго псевдовыпуклых областей теорема 1 есть в точности теорема 1 из [9].

В случае $N=\mathbb{C}$ понятие $K$-предела было впервые введено, по-видимому, А. Кораньи [10] в 1961 г., а различные варианты этого понятия использованы в принадлежащем Е. М. Чирке [11] обобщении на многомерной случай классической теоремы Линделёфа. Автор благодарит рецензента, указавшего ему на эти факты.

\section{СПИСОК ЦИТИРОВАННОЙ ЛИТЕРАТУРЫ}

[1] Royden H. L. Remarks on the Kobayashi metric // Lect. Notes in Math. 1971. V. 185. P. 125-137.

[2] Kobayashi S. Invariant distances on complex manifolds and holomorphic mappings // J. Math. Soc. Japan. 1967. V. 19. № 4. P. 460-480.

[3] Kerzman P., Rosay J.-P. Fonctions plurisubharmoniques d'ehaustion bornées et domaines taut // Math. Ann. 1981. V. 257. №2. P. 171-184.

[4] Kirnan P. On the relations between taut, tight and hyperbolic manifolds // Bull. Amer. Math. Soc. 1970. V. 76. P. 49-51.

[5] Krantz S. G. Invariant metrics and the boundary behavior of holomorphic functions on domains in $\mathbb{C}^{n}$ // J. Geom. Anal. 1991. V. 1. № 2. P. 71-97.

[6] Lehto O., Virtanen K. I. Boundary behaviour and normal meromorphic functions // Acta Math. 1957. V. 97. P. 47-65.

[7] Fefferman C. The Bergman kernel and biholomorphic mappings of pseudoconvex domains // Invent. Math. 1974. V. 26. P. 1-65.

[8] Graham I. Boundary behavior of the Caratheodory and Kobayashi metrics on strongly pseudoconvex domains // Trans. Amer. Math. Soc. 1975. V. 207. P. 219-240.

[9] Довбуш П.В.О существовании допустимых пределов функций многих комплексных переменных // Сиб. матем. ж. 1987. Т. 28. №3. С. 373-377.

[10] Koranyi A. Harmonic functions on Hermitian hyperbolic space // Trans. Amer. Math. Soc. 1969. V. 135. P. 507-516.

[11] Чирка Е. М. Теоремы Линделёфа и Фату в $\mathbb{C}^{n}$ // Матем. сб. 1973. Т. 92. № 4. С. 622-644.

Институт математики и информатики

Поступило

Академии наук Республики Молдова, г. Кишинев

14.03.2003

E-mail: dovbush@math.md

Исправленный вариант

08.07 .2004 